\title{
Monocyte esterase deficiency in malignant neoplasia
}

G M Markey, J A McCormick, T C M Morris, H D Alexander, L Nolan, L M Morgan, $M$ E Reynolds, S Edgar, A L Bell, M D McCaigue, J H Robertson
Belfast City Hospital, Northern Ireland Department of Haematology GM Markey

J A McCormick

T C M Morris

H D Alexander

L Nolan

ME Reynolds

$S$ Edgar

J H Robertson

Laboratory Computer Service

M Morgan

Department of Rheumatology L A Bell

M D McCaigue

Correspondence to: Dr G Markey

Accepted for publication 16 November 1989

\begin{abstract}
A survey of the incidence of monocyte esterase deficiency in 4000 inpatients (including 808 with malignant neoplastic disease) and 474 normal controls was performed using an automated esterase method. A highly significant excess of patients with malignant disease and the deficiency was evident when compared with normal controls or all other patients. Within the group of patients with malignant disease the demonstrable excess occurred in B chronic lymphocytic leukaemia, non-Hodgkin's and Hodgkin's lymphoma, and carcinoma of the gastrointestinal tract. There was also a significant excess of patients with the deficiency attending the renal unit, both among patients who had had renal transplants and those who had not. A familial incidence of monocyte esterase deficiency was found in $19(35 \%)$ of first degree relatives of those patients in whom family studies were done.

It is suggested that the reason for the increased prevalence of the anomaly in these disorders might be that the diminution of esterase activity has a role in their development.
\end{abstract}

Some inheritable enzyme deficiencies have been associated with an increased occurrence of neoplastic disease. Lanza et al described the development of malignant neoplastic disease in $42^{\circ}$ of those with total myeloperoxidase deficiency of polymorphonuclear leucocytes. ${ }^{1}$ Esterase D (an esterase found in most human tissues) deficiency has been shown to occur with chromosomal deletions at $13 \mathrm{q}_{14}{ }^{2}$ and this deletion has been shown to indicate a predisposition to the development of retinoblastoma. ${ }^{3}$ We recently reported an increased occurrence of severe diminution of another esterase-monocyte esterase-in patients with B chronic lymphocytic leukaemia (B-CLL) and non-Hodgkin's lymphoma in a small study sample. ${ }^{4}$ This enzyme diminution was shown to be a familial characteristic with probable autosomal dominant mode of transmission. ${ }^{45}$ This inevitably led to the suspicion that the decrease in monocyte esterase activity might be a causative factor for these diseases or linked to such a factor.

We have now studied a large group of patients attending a regional general hospital-Belfast City Hospital (BCH)-and the Regional Radiotherapy Centre (RCC)-to see if there is an increased prevalence of patients with monocyte esterase deficiency in any other disease group, particularly neoplastic disease groups, and to confirm our previous findings.

\section{Methods}

The study was performed over nine weeks (October, November 1987). The patient populations analysed were from the following units: general surgical, including trauma and vascular $(\mathrm{n}=611)$; urinary surgical $(\mathrm{n}=$ 290); obstetric $(\mathrm{n}=508)$; gynaecological $(\mathrm{n}=$ 198); medical, including respiratory and geriatric $(n=905)$; rheumatological $(n=$ 362 , including 87 samples from the Rheumatology Department of the Royal Victoria Hospital, Belfast); renal $(\mathrm{n}=290)$; haematological $(n=350)$; neonatal $(n=20)$; paediatric $(\mathrm{n}=51)$; oncological $(\mathrm{n}=90)$; Regional Radiotherapy Centre $(n=324)$. The normal samples were from blood donors $(n=474)$.

The total number of patient samples was 6207. A single sample was obtained from $80^{\circ}{ }_{0} ; 20^{\circ}{ }_{0}$ had more than one sample analysed $\left(12^{\circ}{ }_{0}\right.$, two; $4.5 \%$, three; $1.8 \%$, four; $0.8{ }^{\circ}$, five; $1.0 \%$, six or more). From patients with more than one result, the last was accepted.

Records of diagnoses according to the International Classification of Disease of the patients with malignant disease (recorded as primary, or secondary diagnoses on their inpatient charts) who were not in oncology or haematology beds in $\mathrm{BCH}$ during the period of the survey were obtained from the information section of the Eastern Health and Social Services Board of Northern Ireland.

Sequestrene samples received in the $\mathrm{BCH}$ laboratory for routine FBC (or transferred from the RRC) were used for analysis by an automated esterase method when all tests requested had been completed. Only $50 \mu$ l of blood was required.

\section{AUTOMATED ESTERASE METHOD}

The study was carried out using a Hemalog D90, which is an automated cytochemistry analyser for differential white cell counting. It simultaneously counts monocytes on two channels (10000 cells/channel), having stained one aliquot for esterase activity (with $\alpha$ naphthyl butyrate as substrate at $\mathrm{pH} 6 \cdot 1$ ) and another for peroxidase activity. Peroxidase positive but esterase negative monocytes are indicated as a positive remainder value $(+R)$. A low monocyte count on the esterase channel 
can indicate either esterase deficiency of monocytes or a low proportion of monocytes in the sample and cannot be used alone when looking for esterase deficiency in inpatient populations in which decreases or increases in monocyte percentages may occur for several reasons. Therefore a comparison between the proportion of monocytes shown by the esterase channel and that on the peroxidase channel is necessary. The calculation-peroxidase positive esterase negative monocytes $(R) /$ total monocyte count-that is, esterase positive + esterase negative peroxidase positive monocytes; M) $\times 100$ - can be used to find the percentage of esterase negative monocytes. We have previously proved, by manual staining and isoenzyme analysis, that high percentages derived from this calculation correlated well with pronounced monocyte esterase deficiency, except in BCLL where only $50 \%$ of esterase negative identifications were correct. ${ }^{4}$

\section{Definition of pronounced monocyte esterase deficiency}

Only pronounced degrees of enzyme deficiencies are associated with clinical disease. We therefore set a cut off point for the value which defined such a degree of monocyte esterase deficiency. A sample was obtained from a patient with non-Hodgkin's lymphoma who had been identified by the Hemalog $D$ in our previous survey as having pronounced monocyte esterase deficiency. The deficiency was confirmed as described below and by isoenzyme studies, and his father and two siblings (all healthy) were shown to be similarly deficient. This sample was processed 11 times, at intervals on one day at the midpoint of the survey, and the mean (SD) value of esterase negative monocytes shown was 73 $(11)^{\circ}{ }_{0}$. Samples with $\geqslant 73 \%$ esterase negative monocytes were therefore considered to show pronounced monocyte esterase deficiency in this survey.

Samples from four normal esterase positive laboratory staff were analysed 290 times during the course of the survey (about every twentieth sample). On only three occasions were any esterase negative cells present. The highest reading obtained was $36 \%$. Samples from premature infants were used as esterase negative controls whenever available as we were already aware that premature infants have a very high proportion of esterase negative monocytes, the proportion being related to the degree of prematurity. ${ }^{6}$ Forty seven such samples had

$$
\frac{\mathrm{R}}{\mathrm{M}} \times 100 \geqslant 73 \%
$$

during the survey period.

Patients and three of four normal blood donors whose samples had shown the deficiency gave informed consent to the taking of a sample for manual esterase staining. Cytocentrifuge (cytospin) preparations of mononuclear cells (separated over Ficoll-Paque) were made from these samples anticoagulated with heparin (63). Seven sequestrene samples were processed similarly. In 10 cases buffy coat smears were made, and in two patients blood films only were available. Esterase stains using $\alpha$ naphthyl acetate as substrate (ANAE) were performed on batches of these preparations according to the method of Yam et $\mathrm{al}^{7}$ Cytospins of esterase positive monocytes were always included. Lymphocyte dot positivity was provided on slide control for each cytospin. A minimum of 100 monocytes was visualised and monocytes recorded as positive or negative in all cytospin and buffy coat preparations, with the exception of three patients (two with CLL, one with nonHodgkin's lymphoma) whose cytospins showed very few identifiable monocytes. Two of these patients have been recorded as esterase positive because the only identifiable monocytes were positive; the third patient (with CLL) was equivocal as 10 of only 13 monocytes were negative. When only blood films were available the whole esterase stained slide was examined and compared with a Wright stained slide.

Fisher's exact probability test (two-tailed) was used for all comparisons of the prevalence of monocyte esterase deficiency between subgroups of subjects. Spearman's rank correlation coefficient was used to determine whether associations existed between other haematological variables and the percentage of esterase negative monocytes present.

White cell counts were provided for each sample by a Coulter Counter Model $\mathrm{S}+4$ (BCH) or Technicon Hl (RRC). Total monocyte counts were calculated as follows: if no esterase negative peroxidase positive monocytes were recorded by the Hemalog $D$ the monocyte count of that sample was white cell count $\times$ percentage of esterase positive monocytes; if some were present then the monocyte count was white cell count $\times$ percentage of esterase positive monocytes + percentage of esterase negative, peroxidase positive monocytes.

\section{Results}

The 6207 samples analysed yielded results from 4000 patients. Some esterase negative monocytes were present in $799(20 \%)$ of these patients, excluding neonates, and in 40 $(9 \%)$ of the 474 normal blood donors.

\section{MONOCYTE ESTERASE DEFICIENCY}

Table 1 shows the number of patients (excluding premature infants) with and without malignant neoplasia, together with the number of subjects identified with the deficiency. The excess of patients with malignant disease and the deficiency was significant when compared Table 1 Monocyte esterase deficiency in all groups

\begin{tabular}{|c|c|c|}
\hline & $\begin{array}{l}\text { No of } \\
\text { subjects }\end{array}$ & $\begin{array}{l}\text { No }(\%) \text { of subjects } \\
\text { with myocardial } \\
\text { esterase deficiency }\end{array}$ \\
\hline Blood donors & 474 & $4(0.8)$ \\
\hline $\begin{array}{l}\text { Patients with malignant } \\
\text { neoplasia }\end{array}$ & 808 & $32(3.9)$ \\
\hline $\begin{array}{l}\text { Patients with non-malignant } \\
\text { diseases }\end{array}$ & 3172 & $54(1 \cdot 7)$ \\
\hline
\end{tabular}


Table 2 Monocyte esterase deficiency in patients with malignant neoplastic disease, subdivided by disease type

\begin{tabular}{|c|c|c|}
\hline Disease group & $\begin{array}{l}\text { No of } \\
\text { patients }\end{array}$ & $\begin{array}{l}\text { No with monocyte } \\
\text { esterase deficiency }\end{array}$ \\
\hline $\begin{array}{l}\text { Carcinoma: } \\
\text { Gastrointestinal }\end{array}$ & $\begin{array}{r}456 \\
46\end{array}$ & $\begin{array}{l}12(\mathrm{p}=0.0635) \\
51.0 \% \text { to } 19.1 \% \\
(\mathrm{p}<0.001)^{\star}\end{array}$ \\
\hline $\begin{array}{l}\text { Urinary } \\
\text { Breast } \\
\text { Respiratory } \\
\text { Female genital } \\
\text { Others }\end{array}$ & $\begin{array}{r}78 \\
176 \\
67 \\
48 \\
41\end{array}$ & $\begin{array}{l}2 \\
3 \\
1 \\
1 \\
0\end{array}$ \\
\hline Sarcoma & 32 & 0 \\
\hline $\begin{array}{l}\text { Reticuloendothelial } \\
\text { malignancy: }\end{array}$ & 320 & $\begin{array}{c}202.6 \% \text { to } 8.2 \% \\
(p<0.001)^{\star}\end{array}$ \\
\hline $\begin{array}{l}\text { Non-Hodgkin's } \\
\text { lymphoma }\end{array}$ & 113 & $\begin{array}{r}60.3 \% \text { to } 8.7^{\circ} \\
(p=0.0097)^{\star}\end{array}$ \\
\hline BCLL & 36 & $\begin{array}{c}51.7 \% \text { to } 24.4 \% \\
(p<0.001)^{\star}\end{array}$ \\
\hline $\begin{array}{l}\text { Myeloma } \\
\text { Acute lymphoblastic }\end{array}$ & 39 & 2 \\
\hline leukaemia in remission & 10 & 1 \\
\hline Hodgkin's disease & 45 & $\begin{array}{c}40.5 \% \text { to } 17.2 \% \\
(p=0.0018)^{\star}\end{array}$ \\
\hline Myeloproliferative disease & 77 & 2 \\
\hline
\end{tabular}

*Approximate $95^{\circ}$ o confidence limits of difference between normal controls and specified groups.

with that in the non-neoplastic group ( $\mathrm{p}<$ $0.001,95 \%$ confidence limits $0.6 \%$ to $3.9 \%$ ) or the normal controls $(p<0.001,95 \%$ confidence limits $1.5 \%$ to $4.7 \%$ ). In table 2 patients with malignant disease are subdivided according to their type of neoplasia. While the incidence of monocyte esterase deficiency approached significance in patients with carcinoma, in those with carcinoma of the gastrointestinal tract it was significant. In the group with reticuloendothelial neoplasia there was a significant increase in the number of patients with monocyte esterase deficiency in those with BCLL, non-Hodgkin's lymphoma, and Hodgkin's disease.

The patients with carcinoma were further subdivided (table 3 ) according to whether they were in primary diagnostic units-that is, general medical and surgical units in $\mathrm{BCH}(\mathrm{n}=$ 117) - or attending specialist oncological treatment units such as the radiotherapy centre or oncology department $(n=339)$. The incidence of monocyte esterase deficiency in patients attending primary diagnostic units was raised $(\mathrm{p}=0.0112,95 \%$ confidence limits $=2.3 \%$ to $6.3 \%$ ), largely due to a significant excess in patients with gastrointestinal cancer. Patients with this type of cancer attending oncology and radiotherapy units also showed a higher prevalence of monocyte esterase deficiency.
MONOCYTE ESTERASE DEFICIENCY IN PATIENTS WITHOUT CANCER (table 4)

Most premature infants showed monocyte esterase deficiency. Twenty seven per cent of paediatric patients showed the presence of some esterase negative monocytes, although none had monocyte esterase deficiency. Otherwise, significant excess of monocyte esterase deficiency was found only in patients attending the renal unit, although the incidence in patients with rheumatoid arthritis and in autoimmune haematological disease approached significance. In the renal unit monocyte esterase deficiency occurred equally in patients who had had renal transplants and those who had not. The aetiology of the diseases of the patients with monocyte esterase deficiency attending the renal unit was no different from that of all other renal patients. The clinical diagnoses of the 23 patients with monocyte esterase deficiency attending the medical or surgical units showed no apparent specific disease grouping.

\section{CONFIRMATION OF ESTERASE STATE BY MANUAL} STAINING (ANAE)

Material was obtained from 79 of the 86 adult patients with monocyte esterase deficiency and three of the four normal blood donors with the deficiency; no further material could be obtained from seven patients who died (hepatocellular carcinoma, rheumatoid arthritis and pneumonia, perforated bowel), or due to lack of consent (cholelithiasis, appendicitis, hereditary elliptocytosis, drug trial subject). In 50 patients the sample was obtained two to 39 weeks after the original analysis (mean $(\mathrm{SD})=17 \cdot 4(9 \cdot 5)$ ); in 29 it was obtained less than two weeks after the analysis. Sixty four patients had $>85 \%$ esterase negative monocytes; six had $>73 \%$ but $<85 \%$; one was equivocal; and eight showed fewer than $73 \%$. The latter nine patients were considered to have had false identification of monocyte esterase deficiency by the Hemalog $\mathrm{D}$. The diseases of these patients were CLL (one false; one equivocal), non-Hodgkin's lymphoma $(n=1$, white cell count $71 \cdot 7)$, Hodgkin's disease $(n=1)$, chronic renal failure $(n=2)$ (one transplanted), perforated duodenal ulcer $(n=1)$, atheromatous vascular disease and renal failure $(n=1)$, cholestatic jaundice induced by chlorpromazine $(n=1)$. One of the normal donors with

Table 3 Further analysis of patients with malignant neoplastic disease and monocyte esterase deficiency

\begin{tabular}{|c|c|c|c|c|c|c|c|}
\hline Origin of malignancy & Breast & Respiratory & $\begin{array}{l}\text { Gastro- } \\
\text { intestinal }\end{array}$ & Urinary & $\begin{array}{l}\text { Female } \\
\text { genital }\end{array}$ & $\begin{array}{l}\text { Others including } \\
\text { skin and } \\
\text { oropharynx }\end{array}$ & g Unknown \\
\hline \multicolumn{8}{|l|}{$\bar{A}$} \\
\hline $\begin{array}{l}\text { Patients }(n=117) \text { analysed } \\
\text { on Hemalog D }\end{array}$ & 5 & 21 & 28 & 53 & 8 & 2 & 0 \\
\hline $\begin{array}{l}\text { Patients with monocyte } \\
\text { esterase deficiency }\end{array}$ & 1 & 0 & $3 \star$ & 2 & 0 & 0 & 0 \\
\hline \multicolumn{8}{|l|}{$B$} \\
\hline $\begin{array}{l}\text { Patients }(n=339) \text { analysed } \\
\text { on Hemalog D }\end{array}$ & 158 & 45 & 18 & 25 & 34 & 50 & 10 \\
\hline $\begin{array}{l}\text { Patients with monocyte } \\
\text { esterase deficiency }\end{array}$ & 2 & 1 & $2 \dagger$ & 0 & 1 & 0 & 0 \\
\hline
\end{tabular}

$A$ Carcinoma patients attending primary diagnostic (general surgical and medical units $\mathrm{BCH}$ ): prevalence of monocyte esterase deficiency.

$B$ Carcinoma patients attending secondary referral units, (radiotherapy and oncology and haematology): prevalence of monocyte esterase deficiency.

esterase deficiency.

†Approximate $95 \%$ confidence limits $=-4 \cdot 1 \%$ to $24 \cdot 8 \%, \mathrm{p}=0.0348$. 
Table 4 Monocyte esterase deficiency in patients without malignant neoplasia

\begin{tabular}{lcc}
\hline $\begin{array}{l}\text { Units attended } \\
\text { (subspecialities } \\
\text { or disease groups) }\end{array}$ & $\begin{array}{l}\text { No of } \\
\text { patients }\end{array}$ & $\begin{array}{l}\text { No with monocyte } \\
\text { esterase deficiency }\end{array}$ \\
\hline Surgical & 815 & 11 \\
$\quad$ Urinary tract) & $(237)$ & $(3)$ \\
Medical & 882 & 12 \\
(Cardiac) & $(119)$ & $(1)$ \\
Rheumatological & 362 & 9 \\
(Rheumatoid arthritis) & $(218)$ & $(7)(p=0.0547)$ \\
Haematological & 59 & 3 \\
(Autoimmune) & $(22)$ & $(2)(p=0.0506)$ \\
Radiotherapy-Benign & 14 & 0 \\
Renal & 291 & $11(\mathrm{p}=0.0112)^{\star}$ \\
$\quad$ (Renal transplant) & $(131)$ & $(5)$ \\
Obstetric & 508 & 7 \\
Gynaecological & 190 & 1 \\
Paediatric & 51 & 0 \\
Premature baby & 20 & 13 \\
Total & 3192 & 67 \\
\hline
\end{tabular}

$\star 95^{\circ}{ }_{0}$ confidence limits of difference between renal patients and control groups $=0.6^{\circ}{ }_{0}$ to $5 \cdot 3^{\circ}$

monocyte esterase deficiency was esterase positive.

There were 17 patients who had $\geqslant 73 \%$ esterase negative monocytes in some sample before their last sample but not at their last sample analysis. Fourteen of these were shown to be severely esterase deficient by ANAE staining of cytospins. Nine had $85 \%$ esterase negative monocytes: five had $<85 \%$ but $>73^{\circ}{ }_{0}$ esterase negative monocytes. Their spectrum of disease included one with CLL, one with non-Hodgkin's lymphoma (white cell count $82 \cdot 2$ ), one with Hodgkin's disease, two with myeloma, one with bladder carcinoma, one with malignant glioma, four with chronic renal failure (diabetes, Allport's syndrome, ?glomerulonephritis, unknown cause).

There was no significant difference between age distribution and the percentage of esterase negative monocytes in the normal blood donor or any patient groups. Table 5 shows the number of men and women with monocyte esterase deficiency in the blood donor group, all patients with malignant disease, and all patients with non-malignant disorders, excluding premature infants. There was no significant difference in the occurrence of monocyte esterase deficiency between men and women in any group.

In the control group the white cell count did not show a correlation with the percentage of esterase negative monocytes $\left(r_{s}=0.0396\right.$, Spearman's rank correlation coefficient). In the total $\mathrm{BCH}$ group, excluding neonates, there was a significant but slight correlation between these two variables $\left(r_{s}=0.1348, p<0.001\right.$, $95^{\circ}$ o confidence limits $0 \cdot 100$ to $0 \cdot 166$ ) and when the total group of patients with neoplastic

Table 5 Sex distribution of monocyte esterase deficiency

\begin{tabular}{lcc}
\hline Blood donors & Number & $\begin{array}{l}\text { Number with monocyte } \\
\text { esterase deficiency }\end{array}$ \\
\hline Male & 316 & 3 \\
Female & 158 & 1 \\
All patients with malignant disease: & \\
Male & 366 & 18 \\
Female & 442 & 14 \\
All patients with non-malignant disease: & \\
Male & 1111 & 25 \\
Female & 2061 & 29 \\
\hline
\end{tabular}

disease attending haematological, oncological, and radiotherapy units was analysed separately $\left(r_{s}=0.1635, \mathrm{p}<0.001,95^{\circ}\right.$ confidence limits 0.099 to 0.247 ). Analysis of these variables in the groups showing an association with monocyte esterase deficiency did not indicate a significant correlation in any. (CLL, $r_{s}=$ $0 \cdot 1473, p=0.391$; non-Hodgkin's lymphoma, $\mathrm{r}_{s}=0 \cdot 1459, \mathrm{p}=0 \cdot 130 ;$ Hodgkin's disease, $\mathrm{r}_{\mathrm{s}}=$ $0 \cdot 2451, \mathrm{p}=0 \cdot 109$; gastrointestinal tract malignancy, $r_{s}=0.2624, p=0.102$ and renal patients, $r_{s}=0.0495, \mathrm{p}=0.401$ ).

The proportion of esterase negative monocytes present in samples from the normal population showed a significant weak negative correlation with the total monocyte count $\left(r_{s}=\right.$ $-0.3165, p<0.001,95^{\circ}{ }_{0}$ confidence limits -0.396 to -0.232 ). Similar negative correlations were present when total $\mathrm{BCH}\left(\mathbf{r}_{s}=-\right.$ $0.2192, \mathrm{p}<0.001,95^{\circ} \%$ confidence limits -0.252 to $-0 \cdot 186)$ and radiotherapy/oncology groups $r_{s}=-0.0985, p=0.011,95 \%$ confidence limits -0.172 to -0.024$)$ were examined, or when they were divided into subspeciality groups (non-Hodgkin's lymphoma, $r_{s}=-0 \cdot 1243, p=0 \cdot 198$; Hodgkin's disease, $r_{s}=-0.2696, p=0.077$; gastrointestinal cancer $r_{s}=-0.2148, p=0.183$ renal patients, $r_{s}=-0.2594, p<0.001,95 \%$ confidence limits -0.371 to -0.149 ) with the exception of the CLL subgroup in which a weak positive correlation was found $\left(r_{s}=\right.$ $0 \cdot 2419 \mathrm{p}=0 \cdot 155)$.

\section{FAMILY STUDIES}

The families of 11 esterase negative patients in this survey were investigated using the manual technique (CLL, $\mathrm{n}=2$, non-Hodgkin's lymphoma, $\mathrm{n}=3$, rheumatoid arthritis, $\mathrm{n}=3$, vasculitic nephritis, $\mathrm{n}=1$, familial nonAllport's nephritis, $\mathrm{n}=1$, autoimmune haemolytic anaemia and neutropenia, $n=1$ ). Of 54 first degree relatives examined, 19 showed the anomaly $\left(35^{\circ}{ }_{0}\right)$. The figure shows the pedigree of two affected families.
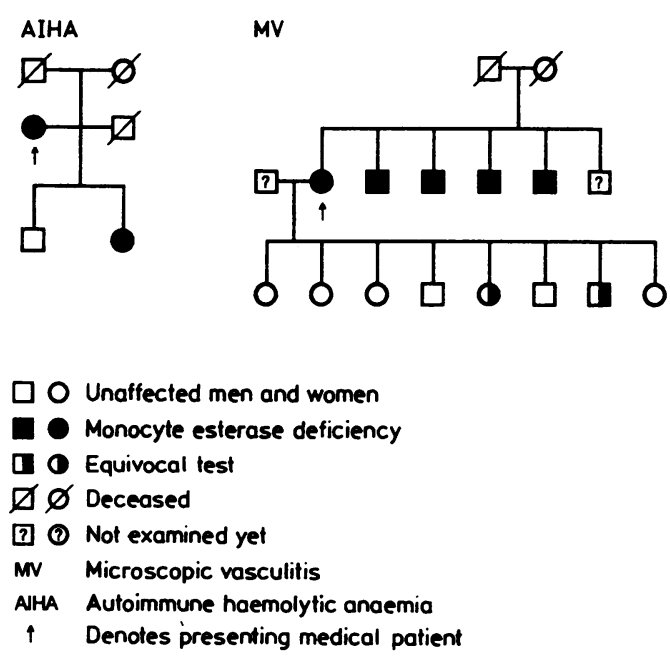

Prevalence of monocyte esterase deficiency in families of two patients with monocyte esterase deficiency identified two patients with
in the survey. 


\section{Discussion}

This study has shown that a higher proportion of patients with certain malignant diseases have monocyte esterase deficiency than the normal population. We have previously shown this in a small study in patients with non-Hodgkin's lymphoma and CLL. The present survey confirms this in a much larger group of patients with these diseases and shows for the first time an increase in the prevalence of monocyte esterase deficiency in patients with Hodgkin's disease and gastrointestinal carcinoma-the latter group showing a significant excess of monocyte esterase deficiency in primary diagnostic units and specialist referral groups. Study of the occurrence of monocyte esterase deficiency in other malignant disease groupsnamely, urinary tract malignancy and breast cancer-in primary diagnostic units suggests that it would be unwise, at this stage, to dismiss an association with them also. Although the means whereby the incidence of the anomaly was surveyed may have been relatively crudde, the examination of large numbers of samples, the identification of the anomaly in only tiny numbers of those samples, the subsequent confirmation by the manual technique, and in addition the previous demonstration of lack of monocyte esterase in similarly identified samples by isoenzyme studies ${ }^{4}$ compensate for this. While there were false positive results, these were matched by failed identifications.

The finding was not an artefact of the instrument because it was subsequently confirmed by manual techniques. The very minor degree of association between the white cell count and percentage of esterase negative monocytes, the negative relation with total monocyte counts, and the absence of monocyte esterase deficiency in most patients with the same diseases, irrespective of their attendance at primary diagnostic units or radiotherapy/ chemotherapy units, show that the increased prevalence of the anomaly in certain disease groups is neither a non-specific result of disease, such as erythrocyte sedimentation rate/C-reactive protein, nor a specific result of certain diseases. Above all else, the high familial incidence of the anomaly, wherever sought, is absolute evidence that the anomaly is not the result of disease or treatment but preceded the occurrence of these diseases.

Why then, should there be an increased prevalence of this anomaly in certain disease groups? If an anomaly occurs at a low level in the normal population and at a higher rate in certain disease groups there can be two basic explanations: (a) that possession of the trait is associated with increased resistance to these diseases, thereby causing an accumulation of affected subjects in these disease groups; or (b) that the trait predisposes to these diseases or is indicative of such a predisposition. The first explanation cannot be dismissed but the spectrum of disease in which we found an increased (neoplastic:renal) or suggestively increased (autoimmune haematological disease:rheumatoid arthritis) incidence of monocyte esterase deficiency in this survey suggests that the latter may be correct. Oertel $e t$ al found that inhibition of monocyte esterase in vitro by bis-nitrophenyl phosphate decreased the ability of the monocyte to lyse K562 cells. ${ }^{8}$ There is, therefore, some experimental evidence to support our hypothesis that severe diminution in monocyte esterase activity, which occurs in an inheritable form and is relatively common, may at least in a minor way, predispose to certain malignant neoplasias or be associated with such a predisposition.

\section{Addendum}

The patient (aged 55), diagnosed with autoimmune haemolytic anaemia and neutropenia (figure) in May 1985 had his spleen removed in July 1987 and presented with carcinoma of the ascending colon in December 1989. He has had a hemicolectomy.

For their help we are very grateful to the medical staff and all the haematology laboratory staff and Mr A Turner of the Belfast City Hospital; the medical laboratory and secretarial staff of the Regional Radiotherapy Centre; the medical and secretarial staff of the oncology uni at the Belfast City Hospital; Drs of the oncology unit at the Belfast City Hospital; Drs M McClelland and C Bharucha and all the staff of the Northern Ireland Blood Transfusion Service; Mr J Robinson of the Department of Health \& Social Services-Information Technology Unit, Belfast and Miss A Graham of the Information Section, Management Services, Eastern Health \& Social Services Board of Northern Ireland; Mr C C Patterson, department of medical statistics, Queen's University and Dr J D Merrett. We also thank Mrs M Ferris for expert secretarial assistance.

The study was made possible by Cancer Research Campaign who also supported Mrs J A McCormick and Mrs M Reynolds.

1 Lanza F, Fietta A, Spisani S, Castoldi GL, Traniello S. Does a relationship exist between neutrophil myeloperoxidase deficiency and the occurrence of neoplasms. J Clin Lab Immunol 1987;22:175-80

2 Sparkes RS, Sparkes ME, Wilson MG, et al. Reginal assignment of genes for human esterase $D$ and retinoblastoma to chromosome band 13q14. Science 1980;208: 1042-4.

3 Wilson MG, Ebbin AJ, Towner JW, Spencer WH Chromosomal anomalies in patients with retinoblastoma. Clin Genet 1979;12:1-8.

4 Markey GM, Morris TCM, Alexander HD. Monocyte esterase? A factor involved in the pathogenesis of lymphoproliferative neoplasia. Leukaemia 1987;1:236-9.

5 Markey GM, Alexander HD, McConnell R, Kyle A, Morris TCM, Robertson JH. Hereditary monocyte esterase deficiency. Br J Haematol 1986;63:359-62.

6 McGuigan EP. Esterase and myeloperoxidase cytochemistry in the neonate and early infant. FIMLS project, University of Ulster, 1987.

7 Yam LT, Li CY, Crosby WH. Cytochemical identification of monocytes and granulocytes. Am J Pathol 1971;55: 283-90.

8 Oertel J, Hagner G, Kastner M, Huhn D. The relevance of $\alpha$ naphthyl acetate esterase to various monocyte functions. Br J Haematol 1985;61:717-26. 


\section{BPMF}

University of London

\section{British Postgraduate Medical Federation \\ Histopathology Course 1990-1991}

There will be a course for SHOs and registrars to provide training in histopathology for the new MRCPath Part 1 examination which begins in May 1992. It will be held on Wednesday afternoons at St Bartholomew's Hospital, and will consist of lectures and slide seminars with emphasis on diagnosis and mechanisms of disease.

The course will run from 3 October 1990-13 March 1991 and is the first in a series of three. The second and third courses will be offered from October 1991 and October 1992 respectively.

Places are restricted and early application is advised.

\section{Cost $£ 517$}

Application forms, which must be returned by 24 August 1990, may be obtained from:

The Education Department, BPMF, 33

Millman Street, London WC1N 3EJ. Telephone 071-831 6222 extension 155

\section{Association of Clinical Pathologists} Junior Membership

Junior membership of the Association is available to medical practitioners who have been engaged in the practice of pathology for a period of less than four years. Junior members are able to remain in this category for a maximum of six years or on the attainment of consultant status. The annual subscription is $£ 24$ for those resident in the United Kingdom and $£ 55$ for those overseas. The annual subscription may be claimed against tax.

Junior members receive the Journal of Clinical Pathology each month. Other benefits are reduced registration fees to attend ACP scientific meetings, all the documents regularly sent to full members of the Association including $A C P$ News, which has a regular column for juniors, and the twice yearly summary of pathology courses included in the ACP programme of postgraduate education. Junior members have their own representative body, the Junior Members' Group, which has a direct input to Council.

For Junior Membership apply to: The Honorary Secretary, Association of Clinical Pathologists, School of Biological Sciences, Falmer, Brighton, BN1 9QG. (0273) 678435.

\section{Second update in cardiopulmonary medicine}

6-8 November, 1990

A three day modular course designed for cardiologists, respiratory physicians, anaesthetics, pathologists, paediatricians and related scientists. Poster sessions will be a feature of the course.

\section{The Squibb Lecture}

Monday 5 November, 1990 at $5.30 \mathrm{pm}$ "Neuroendocrine control of cardiovascular and respiratory systems."

To be given by Professor Julia Polak followed by a champagne reception.

\section{Cardiology Day}

Tuesday 6 November, 1990

Cardiomyopathies-Hypertrophic cardiomyopathies, genetics, cardiomyopathies in fetal life, paediatric cardiomyopathies. Atrioventricular septal defect-Development and structure, deficient atrioventricular septation, atrioventricular conduction tissues, genetics, pulmonary circulation in AVSD.

\section{Cardiopulmonary Day}

Wednesday 7 November, 1990 Endothelin in the heart and lung-Expression in tissue and cell culture, effects: tissue selectivity and vascular activities of endothelin, the heart and coronary circulation, the microvasculature, renal failure, renal function, endothelin in the lung: distribution, receptors and pharmacology, bronchopulmonary actions of endothelin.

\section{Pulmonary Day}

Thursday 8 November, 1990 Cystic fibrosis-Genetics and molecular biology, membrane defect, autonomic innervation and receptor defects, pathological changes, clinical disease spectrum, long term respiratory problems, microbiology and treatment, energy expenditure and nutritional requirements, lung transplantation.

Adult respiratory distress syndromePathology, spectrum of the disease, ventilatory support, electrolyte disturbances, balance and nutrition, cardiovascular assessment and optimising oxygen delivery, recent advances in therapeutic intervention.

\section{Course Fee: $£ 150.00$ whole course} or $\mathbf{6 0 0 . 0 0}$ per day

Further details may be obtained from: The Postgraduate Education Centre, National Heart \& Lung Institute, Dovehouse Street, London SW3 6LY, UK. Tel: 071-351 8172 (24 $\mathrm{hr}$ answering service) Fax: 071-1376 3442
Senior Registrar Management Course, University of Keele

The next management course for senior registrars in pathology directed by Professor Roger Dyson at the University of Keele will be held from lunchtime on Wednesday 12 December, 1990 to lunchtime on Friday 14 December, 1990. If you wish to receive a copy of the programme and booking information for this course, please contact Tanya Matthews, (0782) 621111 ext 3646, or write to the Centre for Health Planning and Management, Suite 2.1, Science Park, University of Keele, Staffs ST5 5SP.

\section{Register of primary immune} deficiencies

In line with other European countries, a Register of all patients in the United Kingdom with primary immune deficiencies is being compiled. This is being organised by $\mathrm{Dr} \mathrm{J}$ Gooi (Immunology Department, Blood Transfusion Service, Bridle Path, Leeds LS15 7TW).

To gain complete coverage we should be grateful if any physicians or general practitioners, who have not already been contacted and who are currently managing such patients, could send details of their patients to Dr Gooi.

Registration forms are available from Dr J Gooi (0532 645091) or Dr H Chapel (0865 817305, Immunology Department, John Radcliffe Hospital, Oxford OX3 9DU).

\section{Corrections}

We regret that an error appeared in the Matters Arising "Screening for bacteriuria" 1989;42:557. The microscopy and culture method was described as costing $£ 0.90$; it should have read $£ 0.09$. Our apologies.

Apologies are extended to Dr G Markey for having changed the gender of the patient reported in his paper 1990;43:282 "Monocyte esterase deficiency in malignant neoplasia". The patient was female.

An error appeared in the last sentence of the paper by Wood et al "Technique for identifying cutting artefacts in sections of undecalcified bone biopsy specimens" 1990;43:516. "This study shows conclusively that areas of fragmentation previously described as bone quality defects are not artefacts which may be created or excluded depending on the plane of the section. They, of course, are artefacts. 